\title{
Sustained Weight Loss over 10 Years in Hypogonadal Men with Type 2 Diabetes Receiving Testosterone in a Registry Study
}

\author{
A Haider ${ }^{1}$, G Doros $^{2}$, KS Haider ${ }^{1}$, A Traish ${ }^{3}$, F Saad $^{4,5}$ \\ 1Private Urology Practice, Bremerhaven, Germany \\ 2Department of Epidemiology and Statistics, Boston University School of Public Health, Boston, MA, USA \\ Department of Biochemistry and Department of Urology, Boston University School of Medicine, Boston, MA, USA \\ ${ }^{4}$ Medical Affairs Andrology, Bayer AG, Berlin, Germany \\ ${ }^{5}$ Gulf Medical University School of Medicine, Ajman, UAE
}

\begin{abstract}
Background
Hypogonadism is highly prevalent in men with type 2 diabetes mellitus (T2DM) as well as obesity and can affect up to $50 \%$ of either condition.

Aim long-term effectiveness and safety of testosterone undecanoate injections (TU) in a subgroup of hypogonadal men from an ongoing registry study in a urological setting.

Aethods registry of hypogonadal men was established in 2004 in a single urologist's office. All men had testosterone $\leq 12.1 \mathrm{nmo} / \mathrm{L}$ diabetologist).

Of these men, 133 received TU $1000 \mathrm{mg} / 12$ weeks (T-group) following an initial 6-week interval. 153 had opted against TTh for various reasons and served as controls (CTRL). Measurements were performed 1-4 times a year for up to 11 years and included, anthropometric parameters, glycaemic control and lipids.

10-year data are reported.

Mean age at baseline was $61.8 \pm 5.4$ years in the T-group and $64.2 \pm 4.6$ years in CTRL.

The majority of men were obese: $91 \%$ in the T-group and $72 \%$ in CTRL. In the T-group, $18 \%$ had excessive obesity, defined by BMI $\geq 40 \mathrm{~kg} / \mathrm{m}^{2}$, in CTRL, $5 \%$. Only 1 patient in the T-group and 5 in CTRL fell into the normal weight category.

Mean weight progressively decreased from $113.1 \pm 13.7$ to $89.6 \pm 9.0 \mathrm{~kg}$ after 10 years in the T-group $(\mathrm{p}<0.0001)$. The decrease was statistically significant vs. previous year for the first 9 years, and then continued without reaching statistical significance. In CTRL, mean weight remained stable from $100.8 \pm 12.9$ to $99.2 \pm 12.9 \mathrm{~kg}$ (NS). The estimated adjusted

difference between groups was $-22.2 \mathrm{~kg}(\mathrm{p}<0.0001)$.

Mean waist circumference progressively decreased from $111.1 \pm 7.4$ to $99.2 \pm 5.5 \mathrm{~cm}$ after 10 years in the T-group $(p<0.0001)$. The decrease was statistically significant vs. previous year for the first 9 years, and then continued without reaching statistica. significance. In CTRL, mean waist circumference fluctuated but dropped during the last 4 years from $115.1 \pm 12.8$ at baseline Mean BMI progressively decreased from $36.2 \pm 4.4$ to $29.1 \pm 2.9 \mathrm{~kg} / \mathrm{m}^{2}$ after 10 years in the T-group $(\mathrm{p}<0.0001)$. The decrease
\end{abstract} was statistically significant vs. previous year for the first 9 years, and then continued without reaching statistical significance.

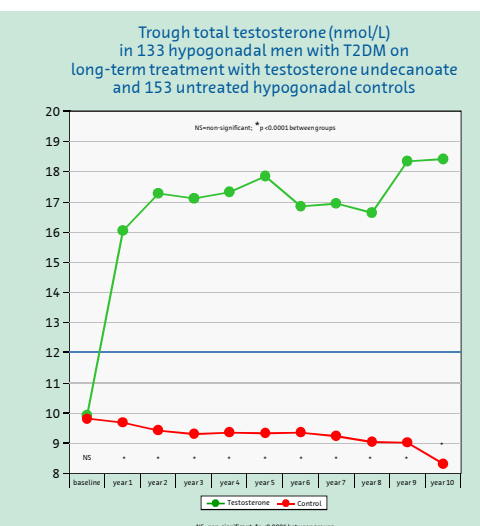

Weight (kg) in 133 hypogonadal men with T2DM on long-term treatment with testosteroneundecanoat d hypogonadal controls

Weight change (\%) in 133 hypogonadal men with T2DM

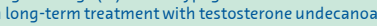
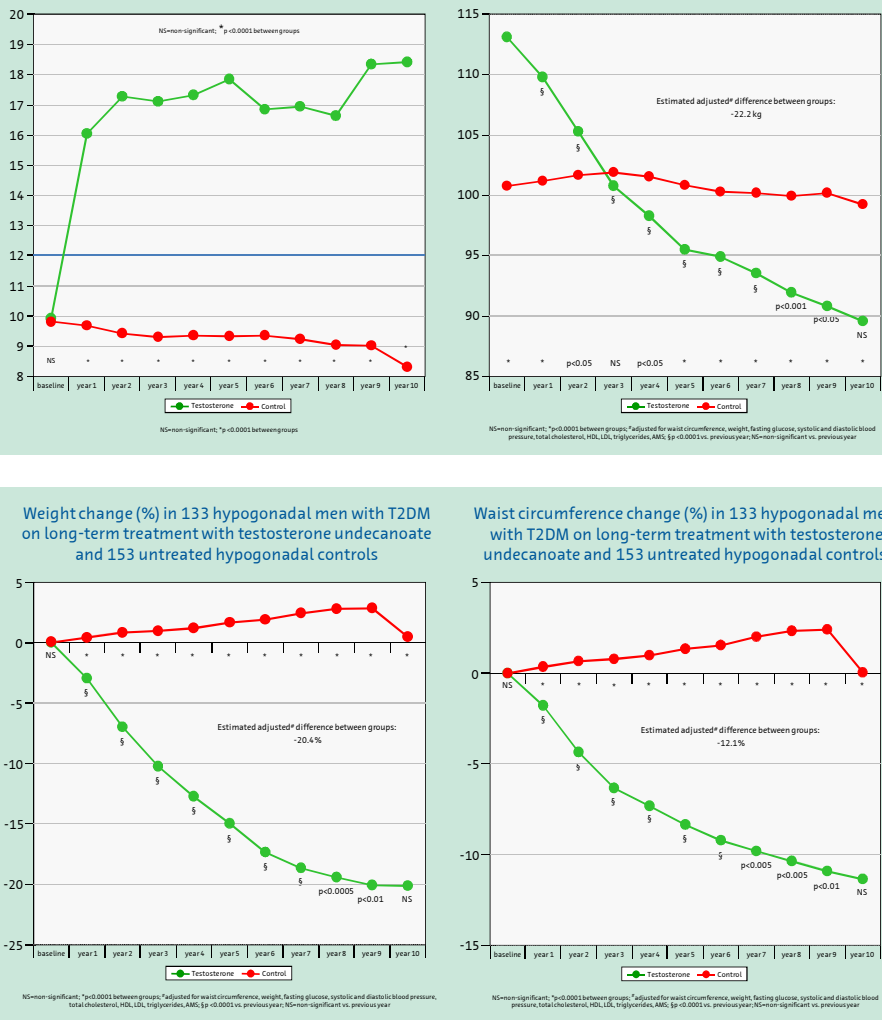

Waist circumference change $(\%)$ in 133 hypogonadal men with T2DM on long-term treatment with testosterone

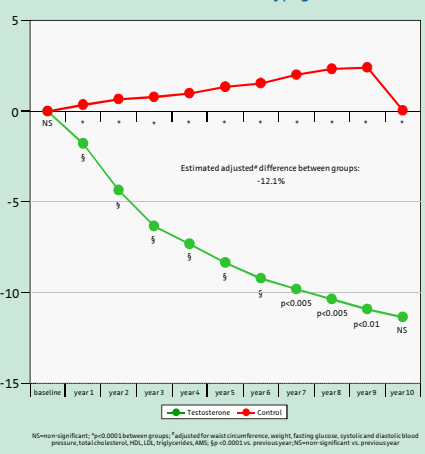

In CTRL, mean BMI remained stable from $32.3 \pm 4.6$ to $32.7 \pm 5.1 \mathrm{~kg} / \mathrm{m}^{2}$ (NS). The estimated adjusted difference between group was $-7.2 \mathrm{~kg} / \mathrm{m}^{2}(\mathrm{p}<0.0001)$.

Per cent weight loss was $-20.1 \pm 5.3 \%$ after 10 years in the T-group ( $p<0.0001$ ). In CTRL, weight change was $0.5 \pm 5.9 \%$ (NS).

Per cent reduction in waist circumference was $-11.4 \pm 3.6 \%$ after 10 years in the T-group $(p<0.0001)$. In CTRL, the adjusted per cent change in waist circumference remained neutral at $0.0 \pm 4.9 \%$ (NS). The estimated adjusted difference between groups

Mean waist:height ratio decreased from $0.63 \pm 0.04$ to $0.57 \pm 0.03$ after 10 years $(p<0.0001)$. The decrease was statistically (NS). The estimated adjusted difference between groups was $-0.08(\mathrm{p}<0.0001)$.

In the T-group, all but 1 patient lost weight, despite the fact that 54 men were on insulin. The only patient who gained $1 \mathrm{~kg}$ of

In the T-group 4 patients $(3 \%)$ died of non-cardiovascuat 12 . (MACE). In CTRL, 24 patients (15.7\%) died. 50 non-fatal MACE occurred (26 myocardial infarctions, 24 strokes). No patient dropped out. Since all injections were administered in the doctor's office and documented, there was a $100 \%$ adher-

Limitations: The study was not designed to investigate effects of testosterone on obesity in men with T2DM. Patients were not randomized as in a medical office setting, neither randomisation nor placebo-control were an option. Patients in the T-group had higher baseline weight while waist circumference at baseline was higher in CTRL.

and close interdisciplinary

Conclusion Long-term treatment with TU in hypogonadal men with T2DM resulted in substantial and sustained weight loss in comparison to untreated contris
instructions.

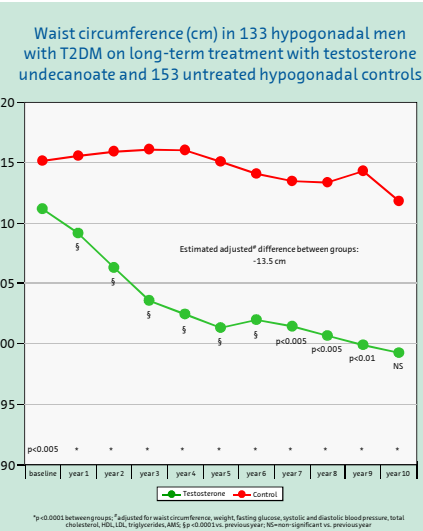

BMI $\left(\mathrm{kg} / \mathrm{m}^{2}\right)$ in 133 hypogonadal men with T2DM

Waist:height ratio in 133 hypogonadal men with $\mathrm{T} 2 \mathrm{DM}$ long-term treatment with testosterone undecanoate and 153 untreated hypogonat controls

Proportion of deaths, Mis and
strokes in T-Group and CTRL

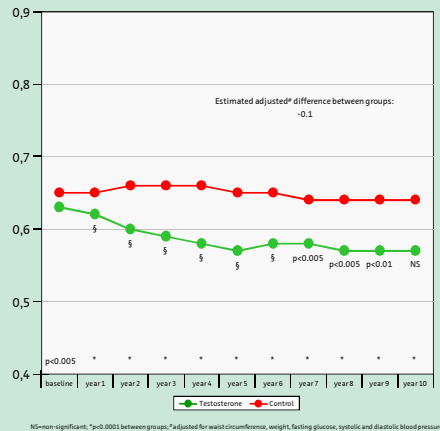

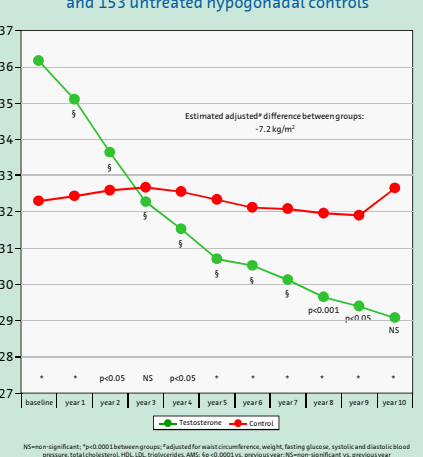

